\title{
Three Topological Indices of Two New Variants of Graph Products
}

\author{
Muhammad Bilal, ${ }^{1}$ Muhammad Kamran Jamil $10,{ }^{1}$ Muhammad Waheed, ${ }^{2}$ \\ and Abdu Alameri $\mathbb{D}^{3}$ \\ ${ }^{1}$ Department of Mathematics, Riphah Institute of Computing and Applied Sciences, Riphah International University, \\ Lahore, Pakistan \\ ${ }^{2}$ Govt Higher Secondary School Nangal Sahdan Muridke, Sheikhupura, Muridke, Pakistan \\ ${ }^{3}$ Department of Biomedical Engineering, University of Science and Technology, Sanaa, Yemen
}

Correspondence should be addressed to Abdu Alameri; a.alameri2222@gmail.com

Received 15 August 2021; Accepted 26 October 2021; Published 19 November 2021

Academic Editor: Mariusz Glabowski

Copyright (C) 2021 Muhammad Bilal et al. This is an open access article distributed under the Creative Commons Attribution License, which permits unrestricted use, distribution, and reproduction in any medium, provided the original work is properly cited.

\begin{abstract}
Graph operations play an important role to constructing complex network structures from simple graphs, and these complex networks play vital roles in different fields such as computer science, chemistry, and social sciences. Computation of topological indices of these complex network structures via graph operation is an important task. In this study, we defined two new variants of graph products, namely, corona join and subdivision vertex join products and investigated exact expressions of the first and second Zagreb indices and first reformulated Zagreb index for these new products.
\end{abstract}

\section{Introduction}

In mathematics, the graph theory is the study of graphs which are mathematical structures used to model pairwise connection between objects. The graph theory is applied in the various fields such as computer science, biology, chemistry, social sciences, and operation research $[1,2]$.

Let $G=(V(G), E(G))$ be a simple, connected graph with vertex set $V(G)$ and edge set $E(G)$. The number of vertices and number of edges are called the order $n$ and size $m$, respectively, of the graph $G$. A graph of order $n$ and size $m$ will be denoted by $G(n, m)$. For any vertex $v \in V(G)$, the degree of vertex $v$ is the number of edges incident on the vertex $v$, and it is written as $d_{G}(v)$ or simply $d(v)$. For a simple graph $G$, the subdivision of the graph $G$ is denoted by $S(G)$ and obtained by inserting a new vertex on every edge of $G$.

Topological index is a numeric value which is associated with a chemical structure of a certain chemical compound. This numeric value can help to predict certain properties of that chemical compound. Hundreds of topological indices have been introduced, but few of them gain attention of the scientific community. Zagreb indices are among the oldest and useful topological indices. For a graph $G$, the first and second Zagreb indices are defined as

$$
\begin{aligned}
& M_{1}(G)=\sum_{v \in V(G)} d_{G}(v)^{2}=\sum_{u v \in E(G)}\left[d_{G}(u)+d_{G}(v)\right], \\
& M_{2}(G)=\sum_{u v \in E(G)} d_{G}(u) d_{G}(v) .
\end{aligned}
$$

In 1972, these topological indices were applied for the first time to find the total electron $\pi$-energy of molecular graphs [3]. Later, the Zagreb indices developed important applications in QSPR/QSAR studies, and a lot of research studies have been published on these [4-10].

Milićević et al. in 2004 reformulated the Zagreb indices in terms of edge degree which is defined as

$$
\begin{aligned}
& E M_{1}(G)=\sum_{e \in E(G)} d(e)^{2}, \\
& E M_{2}(G)=\sum_{e \sim f} d(e) d(f),
\end{aligned}
$$


where $d(e)$ shows the degree of the edge $e$ in $G$, which is defined as $d(e)=d(u)+d(v)-2$ with edge $e=u v$ and $e \sim f$ shows that the edge $e$ and $f$ are adjacent [11].

Graph operations, especially graph products, play a significant role not only in pure and applied mathematics but also in computer science, chemistry, electrical engineering, and pharmaceutics. For instance, the Cartesian product provides a significant model for connecting computers [12].

Let $G_{1}\left(n_{1}, m_{1}\right)$ and $G_{2}\left(n_{2}, m_{2}\right)$ be two connected simple graphs. Corona product of graphs $G_{1}$ and $G_{2}$, denoted by $G_{1}{ }^{\circ} G_{2}$, is obtained by taking one copy of $G_{1}$ and $n_{1}$ copies of $G_{2}$, and joining each vertex of $i^{\text {th }}$ copy of $G_{2}$ to $i^{\text {th }}$ vertex of $G_{1}$ [8]. In [13], authors introduced two variants of the corona product and discussed their spectral properties. The subdivision vertex variant of corona of $G_{1}$ and $G_{2}$ is attained from $S\left(G_{1}\right)$ and $n_{1}$ copies of $G_{2}$ by joining the $i^{\text {th }}$ vertex of $V\left(G_{1}\right)$ to every vertex in the $i^{\text {th }}$ copy of $G_{2}$. Similarly, the subdivision-edge neighborhood corona is obtained by attaching the neighbors of the $i^{\text {th }}$ vertex of $V\left(G_{1}\right)$ to every vertex in the $i^{\text {th }}$ copy of $G_{2}$.

The join graph of $G_{1}$ and $G_{2}$ is obtained by joining each vertex of $G_{1}$ to each vertex of $G_{2}$, and it is denoted by $G_{1}+G_{2}$ [14].

Khalifeh et al. [15] computed the first and second Zagreb indices of Cartesian product, composition, join, disjunction, and symmetric difference of graphs and applied the results on $C_{4}$ tube, torus, and multiwalled polyhex nanotorus. Authors in [16] investigated the upper bounds on the multiplicative Zagreb indices of some product of graphs. Azari and Iranmanesh [17] discussed the rooted product of graphs and found the exact expression of first and second Zagreb indices for this product. Jamil and Tomescu [18] found the exact formulas of the first reformulated Zagreb index for Cartesian product, composition, join, corona product, splice, link, and chain of graphs. Some graph operations and their topological indices are presented in [13, 15-28].

Now, we define variants of these graphs' product.

Definition 1. Let $G_{1}\left(n_{1}, m_{1}\right)$ and $G_{2}\left(n_{2}, m_{2}\right)$ be simple connected graphs, and the corona join graph of $G_{1}$ and $G_{2}$ is obtained by taking one copy of $G_{1}, n_{1}$ copies of $G_{2}$, and joining each vertex of the $i^{\text {th }}$ copy of $G_{2}$ with all vertices of $G_{1}$. The corona join product of $G_{1}$ and $G_{2}$ is denoted by $G_{1} \oplus G_{2}$ and shown in Figure 1 .

Definition 2. For $G_{1}\left(n_{1}, m_{1}\right)$ and $G_{2}\left(n_{2}, m_{2}\right)$, the subdivision vertex join is denoted by $G_{1} \dot{+} G_{2}$ and obtained by joining the each new vertex of $S\left(G_{1}\right)$ to all vertices of $G_{2}$. Figure 2 shows the illustration of subdivision vertex join for $P_{3}$ and $P_{2}$.

\section{Main Results}

In this section, we present the main results. The following lemmas are useful to obtain the exact expressions of topological indices of new variants of graph products. The proofs of the following two lemmas are directly from the definitions of corona join product $G_{1} \oplus G_{2}$ and subdivision vertex join $G_{1} \dot{+} G_{2}$.

Lemma 1. Let $G_{1}=\left(n_{1}, m_{1}\right)$ and $G_{2}=\left(n_{2}, m_{2}\right)$ be two graphs; then, the degree behavior of vertices in the graph $G_{1} \oplus G_{2}$ is

$$
d_{G_{1} \oplus G_{2}}(v)= \begin{cases}d_{G_{1}}(v)+n_{1} n_{2}, & \text { if } v \in V\left(G_{1}\right), \\ d_{G_{2}}(v)+n_{1}, & \text { if } v \in V\left(G_{2}\right) .\end{cases}
$$

Lemma 2. Let we have three simple connected graphs $G_{1}=$ $\left(n_{1}, m_{1}\right)$ and $S\left(G_{1}\right)=\left(n_{1}^{\prime}, m_{1}^{\prime}\right)$; then, the degree behavior of vertices in the graph $G_{1} \dot{+} G_{2}$ is

$$
d_{G_{1}+G_{2}}(v)= \begin{cases}d_{G_{1}}(v), & \text { if } v \in V\left(G_{1}\right), \\ 2+n_{2}, & \text { if } v \in V_{s}\left(G_{1}\right), \\ d_{G_{2}}(v)+m_{1}, & \text { if } v \in V\left(G_{2}\right) .\end{cases}
$$

The following result gives the formula of the first Zagreb index for $G_{1} \oplus G_{2}$.

Theorem 1. Let $G_{1}\left(n_{1}, m_{1}\right)$ and $G_{2}\left(n_{2}, m_{2}\right)$ be two simple graphs; then, the first Zagreb index of corona join product $G_{1} \oplus G_{2}$ is given as

$$
M_{1}\left(G_{1} \oplus G_{2}\right)=M_{1}\left(G_{1}\right)+n_{1} M_{1}\left(G_{2}\right)+n_{1}^{3} n_{2}\left(n_{2}+1\right)+4 n_{1}\left(m_{1} n_{2}+m_{2} n_{1}\right)
$$

Proof. From the definition of the first Zagreb index, we have

$$
M_{1}\left(G_{1} \oplus G_{2}\right)=\sum_{v \in V\left(G_{1} \oplus G_{2}\right)} d_{G_{1} \oplus G_{2}}(v)^{2} .
$$



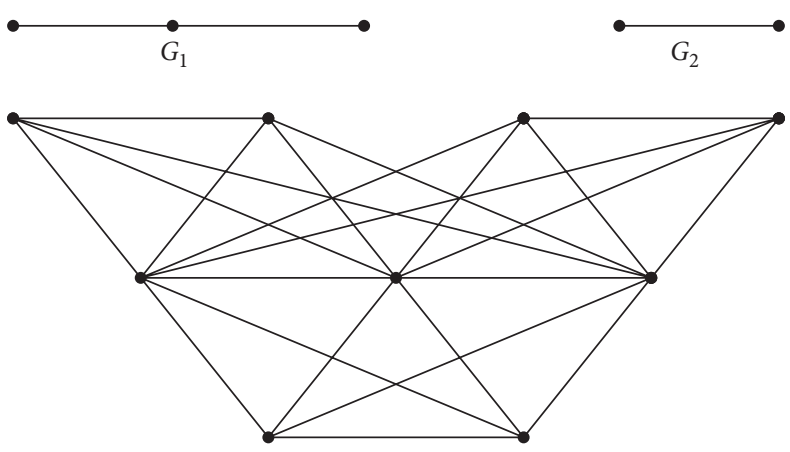

Figure 1: Corona join product $G_{1} \oplus G_{2}$.
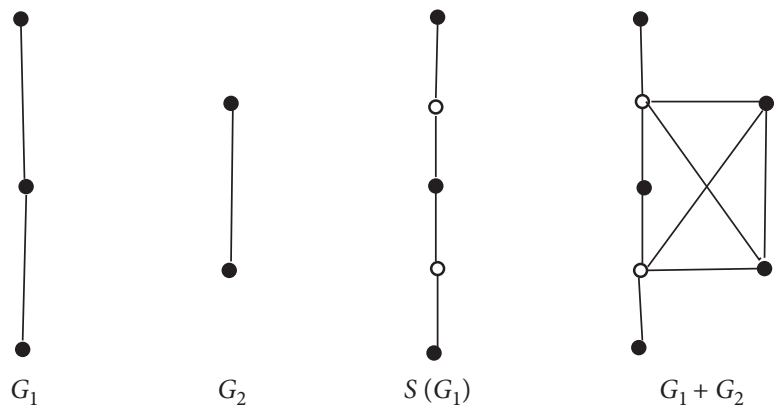

Figure 2: Subdivision vertex join graph $G_{1} \dot{+} G_{2}$.

Now, we apply Lemma 1:

$$
\begin{aligned}
= & \sum_{v \in V\left(G_{1}\right)}\left(d_{G_{1}}(v)+n_{1} n_{2}\right)^{2}+\sum_{v \in V\left(G_{1}\right)} \sum_{v \in V\left(G_{2}\right)}\left(d_{G_{2}}(v)+n_{1}\right)^{2} \\
= & \sum_{v \in V\left(G_{1}\right)}\left(d_{G_{1}}(v)^{2}+\left(n_{1} n_{2}\right)^{2}+2 d_{G_{1}}(v)\left(n_{1} n_{2}\right)\right)+ \\
& \sum_{v \in V\left(G_{1}\right)} \sum_{v \in V\left(G_{2}\right)}\left(d_{G_{2}}(v)^{2}+n_{1}^{2}+2 d_{G_{2}}(v)\left(n_{1}\right)\right) \\
= & \sum_{v \in V\left(G_{1}\right)} d_{G_{1}}(v)^{2}+\left(n_{1} n_{2}\right)^{2} \sum_{v \in V\left(G_{1}\right)} 1+2\left(n_{1} n_{2}\right) \sum_{v \in V\left(G_{1}\right)} d_{G_{1}}(v)+\sum_{v \in V\left(G_{1}\right)} 1 \\
& \sum_{v \in V\left(G_{2}\right)} d_{G_{2}}(v)^{2}+n_{1}^{2} \sum_{v \in V\left(G_{1}\right)} 1 \sum_{v \in V\left(G_{2}\right)} 1+2 n_{1} \sum_{v \in V\left(G_{1}\right)} 1 \sum_{v \in V\left(G_{2}\right)} d_{G_{2}}(v) \\
= & M_{1}\left(G_{1}\right)+n_{1}^{3} n_{2}^{2}+4 m_{1} n_{1} n_{2}+n_{1} M_{1}\left(G_{2}\right)+n_{1}^{3} n_{2}+4 m_{2} n_{1}^{2} \\
= & M_{1}\left(G_{1}\right)+n_{1} M_{1}\left(G_{2}\right)+n_{1}^{3} n_{2}\left(n_{2}+1\right)+4 n_{1}\left(m_{1} n_{2}+m_{2} n_{1}\right),
\end{aligned}
$$

which is our required result.

The next theorem is about the exact expression of the second Zagreb index for $G_{1} \oplus G_{2}$.

Theorem 2. For simple graphs $G_{1}\left(n_{1}, m_{1}\right)$ and $G_{2}\left(n_{2}, m_{2}\right)$, the second Zagreb index of corona join product $G_{1} \oplus G_{2}$ is given as

$$
\begin{aligned}
M_{2}\left(G_{1} \oplus G_{2}\right)= & n_{1}\left[n_{2} M_{1}\left(G_{1}\right)+n_{1} M_{1}\left(G_{2}\right)\right] \\
& +M_{2}\left(G_{1}\right)+n_{1} M_{2}\left(G_{2}\right)+m_{2} n_{1}\left(n_{1}^{2}+4 m_{1}\right) \\
& +n_{1}^{2} n_{2}\left[m_{1} n_{2}+2 m_{1}+2 m_{2} n_{1}+n_{1}^{2} n_{2}\right] .
\end{aligned}
$$

Proof. From the definition of the second Zagreb index, we have 


$$
M_{2}\left(G_{1} \oplus G_{2}\right)=\sum_{u v \in E\left(G_{1} \oplus G_{2}\right)} d_{G_{1} \oplus G_{2}}(u) \cdot d_{G_{1} \oplus G_{2}}(v) .
$$

Now, we apply the Lemma 1:

$$
\begin{aligned}
= & \sum_{u v \in E\left(G_{1}\right)}\left(d_{G_{1}}(u)+n_{1} n_{2}\right)\left(d_{G_{1}}(v)+n_{1} n_{2}\right) \\
& +\sum_{v \in V\left(G_{1}\right)} \sum_{u v \in E\left(G_{2}\right)}\left(d_{G_{2}}(u)+n_{1}\right)\left(d_{G_{2}}(v)+n_{1}\right) \\
+ & \sum_{v \in V\left(G_{1}\right)} \sum_{u v \in E\left(G_{1} \oplus G_{2}\right) u \in V\left(G_{1}\right) v \in V\left(G_{2}\right)}\left(d_{G_{1}}(u)+n_{1} n_{2}\right) \\
& \left(d_{G_{2}}(v)+n_{1}\right) \\
= & \sum_{u v \in E\left(G_{1}\right)} d_{G_{1}}(u) d_{G_{1}}(v)+n_{1} n_{2} \sum_{u v \in E\left(G_{1}\right)}\left[d_{G_{1}}(u)+d_{G_{1}}(v)\right]+\left(n_{1} n_{2}\right)^{2} \\
& \sum_{u v \in E\left(G_{1}\right)} 1+n_{1} \sum_{u v \in E\left(G_{2}\right)} d_{G_{2}}(u) d_{G_{2}}(v)+n_{1}^{2} \sum_{u v \in E\left(G_{2}\right)}\left[d_{G_{2}}(u)+d_{G_{2}}(v)\right]+n_{1}^{3} \\
& \sum_{u v \in E\left(G_{2}\right)} 1+n_{1} \sum_{u \in V\left(G_{1}\right)} d_{G_{1}}(u) \sum_{v \in V\left(G_{2}\right)} d_{G_{2}}(v)+n_{1}^{2} \sum_{u \in V\left(G_{1}\right)} d_{G_{1}}(u) \sum_{v \in V\left(G_{2}\right)} 1+n_{1}^{2} n_{2} \quad \sum d_{v \in V\left(G_{2}\right)} d_{G_{2}}(v) \\
& \sum_{u \in V\left(G_{1}\right)} 1+n_{1}^{3} n_{2} \sum_{u \in V\left(G_{1}\right)} \sum_{v \in V\left(G_{2}\right)} i_{1} \\
= & M_{2}\left(G_{1}\right)+n_{1} n_{2} M_{1}\left(G_{1}\right)+m_{1} n_{1}^{2} n_{2}^{2}+n_{1} M_{2}\left(G_{2}\right)+n_{1}^{2} M_{1}\left(G_{2}\right)+m_{2} n_{1}^{3} \\
+ & 4 m_{1} m_{2} n_{1}+2 n_{1}^{2} n_{2} m_{1}+2 n_{1}^{3} n_{2} m_{2}+n_{1}^{4} n_{2}^{2} \\
= & n_{1}\left[n_{2} M_{1}\left(G_{1}\right)+n_{1} M_{1}\left(G_{2}\right)\right]+M_{2}\left(G_{1}\right)+n_{1} M_{2}\left(G_{2}\right)+m_{2} n_{1}\left(n_{1}^{2}+4 m_{1}\right)+ \\
& n_{1}^{2} n_{2}\left[m_{1} n_{2}+2 m_{1}+2 m_{2} n_{1}+n_{1}^{2} n_{2}\right],
\end{aligned}
$$

which is our required result.

Theorem 3. Let $G_{1}=\left(n_{1}, m_{1}\right)$ and $G_{2}=\left(n_{2}, m_{2}\right)$ be two simple graphs; then, the first reformulated Zagreb index of corona join product $G_{1} \oplus G_{2}$ is

$$
\begin{aligned}
& E M_{1}\left(G_{1} \oplus G_{2}\right) \\
& \quad=5 n_{1}\left[n_{2} M_{1}\left(G_{1}\right)+n_{1} M_{1}\left(G_{2}\right)\right]+E M_{1}\left(G_{1}\right)+n_{1}\left[E M_{1}\left(G_{2}\right)+8 m_{1} m_{2}\right] \\
& \quad+4 m_{1} n_{1} n_{2}\left(n_{1} n_{2}-2\right)+4 m_{2} n_{1}^{2}\left(n_{1}-2\right)+\left(n_{1}+n_{1} n_{2}-2\right) \\
& {\left[4 m_{1} n_{1} n_{2}+4 m_{2} n_{1}^{2}+n_{1}^{2} n_{2}\left(n_{1}+n_{1} n_{2}-2\right)\right] .}
\end{aligned}
$$

Proof. From the definition of the first reformulated Zagreb index and by Lemma 1 , we have 


$$
\begin{aligned}
& E M_{1}\left(G_{1} \oplus G_{2}\right)=\sum_{u v \in E\left(G_{1} \oplus G_{2}\right)}\left[d_{G_{1} \oplus G_{2}}(u)+d_{G_{1} \oplus G_{2}}(v)-2\right]^{2} \\
& =\sum_{u v \in E\left(G_{1}\right)}\left[d_{G_{1}}(u)+n_{1} n_{2}+d_{G_{1}}(v)+n_{1} n_{2}-2\right]^{2}+n_{1} \sum_{u v \in E\left(G_{2}\right)}\left[d_{G_{2}}(u)+n_{1}+d_{G_{2}}(v)+n_{1}-2\right]^{2} \\
& +n_{1} \sum_{u v \in E\left(G_{1} \oplus G_{2}\right)}\left[d_{G_{1}}(u)+n_{1} n_{2}+d_{G_{2}}(v)+n_{1}-2\right]^{2} \\
& u \in V\left(G_{1}\right) \\
& v \in V\left(G_{2}\right) \\
& =\sum_{u v \in E\left(G_{1}\right)}\left[\left(d_{G_{1}}(u)+d_{G_{1}}(v)-2\right)+2 n_{1} n_{2}\right]^{2}+n_{1} \sum_{u v \in E\left(G_{2}\right)}\left[\left(d_{G_{2}}(u)+d_{G_{2}}(v)-2\right)+2 n_{1}\right]^{2} \\
& +n_{1} \sum_{u v \in E\left(G_{1} \oplus G_{2}\right)}\left[\left(d_{G_{1}}(u)+d_{G_{2}}(v)\right)+\left(n_{1}+n_{1} n_{2}-2\right)\right]^{2} \\
& u \in V\left(G_{1}\right) \\
& v \in V\left(G_{2}\right) \\
& =\sum_{u v \in E\left(G_{1}\right)}\left[\left(d_{G_{1}}(u)+d_{G_{1}}(v)-2\right)^{2}+4 n_{1}^{2} n_{2}^{2}+4 n_{1} n_{2}\left(d_{G_{1}}(u)+d_{G_{1}}(v)-2\right)\right] \\
& +n_{1} \sum_{u v \in E\left(G_{2}\right)}\left[\left(d_{G_{2}}(u)+d_{G_{2}}(v)-2\right)^{2}+4 n_{1}^{2}+4 n_{1}\left(d_{G_{2}}(u)+d_{G_{2}}(v)-2\right)\right] \\
& +n_{1} \sum_{u v \in E\left(G_{1} \oplus G_{2}\right)}\left[\left(d_{G_{1}}(u)+d_{G_{2}}(v)\right)^{2}+\left(n_{1}\right) v \in V\left(G_{1} n_{2}-2\right)^{2}+2\left(n_{1}+n_{1} n_{2}-2\right)\right. \\
& \left(d_{G_{1}}(u)+d_{G_{2}}(v)\right) \\
& =\sum_{u v \in E\left(G_{1}\right)}\left(d_{G_{1}}(u)+d_{G_{1}}(v)-2\right)^{2}+4 n_{1}^{2} n_{2}^{2} \sum_{u v \in E\left(G_{1}\right)} 1+4 n_{1} n_{2} \sum_{u v \in E\left(G_{1}\right)}\left(d_{G_{1}}(u)+d_{G_{1}}(v)\right) \\
& -8 n_{1} n_{2} \sum_{u v \in E\left(G_{1}\right)} 1+n_{1} \sum_{u v \in E\left(G_{2}\right)}\left(d_{G_{2}}(u)+d_{G_{2}}(v)-2\right)^{2}+4 n_{1}^{3} \sum_{u v \in E\left(G_{2}\right)} 1 \\
& +4 n_{1}^{2} \sum_{u v \in E\left(G_{2}\right)}\left(d_{G_{2}}(u)+d_{G_{2}}(v)\right)-8 n_{1}^{2} \sum_{u v \in E\left(G_{2}\right)} 1+n_{1} \sum_{\substack{u v \in E\left(G_{1} \oplus G_{2}\right) \\
u \in V\left(G_{1}\right)}}\left(d_{G_{1}}(u)+d_{G_{2}}(v)\right)^{2} \\
& v \in V\left(G_{2}\right) \\
& +n_{1}\left(n_{1}+n_{1} n_{2}-2\right)^{2} \sum_{\substack{u v \in E\left(G_{1} \oplus G_{2}\right) \\
u \in V\left(G_{1}\right) \\
v \in V\left(G_{2}\right)}} 1+2 n_{1}\left(n_{1}+n_{1} n_{2}-2\right) \sum_{\substack{u v \in E\left(G_{1} \oplus G_{2}\right) \\
u \in V\left(G_{1}\right) \\
v \in V\left(G_{2}\right)}}\left(d_{G_{1}}(u)+d_{G_{2}}(v)\right) \\
& =\sum_{u v \in E\left(G_{1}\right)}\left(d_{G_{1}}(u)+d_{G_{1}}(v)-2\right)^{2}+4 n_{1}^{2} n_{2}^{2} \sum_{u v \in E\left(G_{1}\right)} 1+4 n_{1} n_{2} \sum_{u v \in E\left(G_{1}\right)}\left(d_{G_{1}}(u)+d_{G_{1}}(v)\right) \\
& -8 n_{1} n_{2} \sum_{u v \in E\left(G_{1}\right)} 1+n_{1} \sum_{u v \in E\left(G_{2}\right)}\left(d_{G_{2}}(u)+d_{G_{2}}(v)-2\right)^{2}+4 n_{1}^{3} \sum_{u v \in E\left(G_{2}\right)} 1 \\
& +4 n_{1}^{2} \sum_{u v \in E\left(G_{2}\right)}\left(d_{G_{2}}(u)+d_{G_{2}}(v)\right)-8 n_{1}^{2} \sum_{u v \in E\left(G_{2}\right)} 1+n_{1} \sum_{u \in V\left(G_{1}\right)} d_{G_{1}}(u)^{2} \sum_{v \in V\left(G_{2}\right)} 1+ \\
& n_{1} \sum_{v \in V\left(G_{2}\right)} d_{G_{2}}(v)^{2} \sum_{u \in V\left(G_{1}\right)} 1+2 n_{1} \sum_{u \in V\left(G_{1}\right)} d_{G_{1}}(u) \sum_{v \in V\left(G_{2}\right)} d_{G_{2}}(v)+n_{1}\left(n_{1}+n_{1} n_{2}-2\right)^{2} \\
& \sum_{u \in V\left(G_{1}\right)} 1 \sum_{v \in V\left(G_{2}\right)} 1+2 n_{1}\left(n_{1}+n_{1} n_{2}-2\right) \sum_{u \in V\left(G_{1}\right)} d_{G_{1}}(u) \sum_{v \in V\left(G_{2}\right)} 1+2 n_{1}\left(n_{1}+n_{1} n_{2}-2\right) \\
& \sum_{v \in V\left(G_{2}\right)} d_{G_{2}}(v) \sum_{u \in V\left(G_{1}\right)} 1
\end{aligned}
$$




$$
\begin{aligned}
= & E M_{1}\left(G_{1}\right)+4 n_{1}^{2} n_{2}^{2} m_{1}+4 n_{1} n_{2} M_{1}\left(G_{1}\right)-8 n_{1} n_{2} m_{1}+n_{1} E M_{1}\left(G_{1}\right)+4 n_{1}^{3} m_{2} \\
& +4 n_{1}^{2} M_{1}\left(G_{2}\right)-8 n_{1}^{2} m_{2}+n_{1} n_{2} M_{1}\left(G_{1}\right)+n_{1}^{2} M_{1}\left(G_{2}\right)+8 m_{1} m_{2} n_{1}+n_{1}^{2} n_{2}\left(n_{1}+n_{1} n_{2}-2\right)^{2} \\
+ & 4 m_{1} n_{1} n_{2}\left(n_{1}+n_{1} n_{2}-2\right)+4 m_{2} n_{1}^{2}\left(n_{1}+n_{1} n_{2}-2\right) \\
= & 5 n_{1}\left[n_{2} M_{1}\left(G_{1}\right)+n_{1} M_{1}\left(G_{2}\right)\right]+E M_{1}\left(G_{1}\right)+n_{1}\left[E M_{1}\left(G_{2}\right)+8 m_{1} m_{2}\right] \\
& +4 m_{1} n_{1} n_{2}\left(n_{1} n_{2}-2\right)+4 m_{2} n_{1}^{2}\left(n_{1}-2\right)+\left(n_{1}+n_{1} n_{2}-2\right) \\
& {\left[4 m_{1} n_{1} n_{2}+4 m_{2} n_{1}^{2}+n_{1}^{2} n_{2}\left(n_{1}+n_{1} n_{2}-2\right)\right], }
\end{aligned}
$$

hence the required result.

In the following results, we discussed the Zagreb indices of the subdivision vertex join graph.
Theorem 4. For simple graphs $G_{1}=\left(n_{1}, m_{1}\right)$ and $G_{2}=\left(n_{2}, m_{2}\right)$, the first Zagreb index of subdivision vertex join $G_{1}+G_{2}$ is given as

$$
M_{1}\left(G_{1} \dot{+} G_{2}\right)=M_{1}\left(G_{1}\right)+M_{1}\left(G_{2}\right)+n_{2}\left(n_{2}+2\right)^{2}+4 m_{1} m_{2}+n_{2} m_{1}^{2}
$$

Proof. Applying Lemma 2 in the definition of first Zagreb index, we have the following:

$$
\begin{aligned}
M_{1}\left(G_{1} \dot{+} G_{2}\right)= & \sum_{v \in V\left(G_{1}+G_{2}\right)} d_{G_{1}+G_{2}}(v)^{2} \\
= & \sum_{v \in V\left(G_{1}\right)} d_{G_{1}}(v)^{2}+\sum_{v \in V_{s}\left(G_{1}\right)}\left(2+n_{2}\right)^{2}+\sum_{v \in V\left(G_{2}\right)}\left(d_{G_{2}}(v)+m_{1}\right)^{2} \\
= & \sum_{v \in V\left(G_{1}\right)} d_{G_{1}}(v)^{2}+\sum_{v \in V_{s}\left(G_{1}\right)}\left(2+n_{2}\right)^{2}+\sum_{v \in V\left(G_{2}\right)}\left(d_{G_{2}}(v)^{2}+m_{1}^{2}+2 m_{1} d_{G_{2}}(v)\right) \\
= & \sum_{v \in V\left(G_{1}\right)} d_{G_{1}}(v)^{2}+\left(2+n_{2}\right)^{2} \sum_{v \in V_{s}\left(G_{1}\right)} 1+\sum_{v \in V\left(G_{2}\right)} d_{G_{2}}(v)^{2}+m_{1}^{2} \sum_{v \in V\left(G_{2}\right)} 1 \\
& +2 m_{1} \sum_{v \in V\left(G_{2}\right)} d_{G_{2}}(v) \\
= & M_{1}\left(G_{1}\right)+m_{1}\left(n_{2}+2\right)^{2}+M_{1}\left(G_{2}\right)+n_{2} m_{1}^{2}+4 m_{1} m_{2} \\
= & M_{1}\left(G_{1}\right)+M_{1}\left(G_{2}\right)+m_{1}\left(n_{2}+2\right)^{2}+4 m_{1} m_{2}+n_{2} m_{1}^{2},
\end{aligned}
$$

which is our required result.

$$
\begin{aligned}
& M_{2}\left(G_{1} \dot{+} G_{2}\right)= \\
& m_{1} M_{1}\left(G_{2}\right)+M_{2}\left(G_{2}\right)+m_{1}^{2} m_{2}+m_{1}\left(2+n_{2}\right)\left(2 m_{2}+m_{1} n_{2}\right) \\
& \quad+\left(2+n_{2}\right) \sum_{u v \in E\left(S\left(G_{1}\right)\right)} d_{G_{1}}(u) .
\end{aligned}
$$

Theorem 5. Let $G_{1}=\left(n_{1}, m_{1}\right)$ and $G_{2}=\left(n_{2}, m_{2}\right)$ are two simple graphs and $G_{1} \dot{+} G_{2}$ be their subdivision vertex join graph; then, the second Zagreb index of $G_{1}+G_{2}$ is given as 
Proof. From the definition of the second Zagreb index, we Now, we apply Lemma 2: have

$$
M_{2}\left(G_{1} \dot{+} G_{2}\right)=\sum_{u v \in E\left(G_{1}+G_{2}\right)} d_{G_{1}+G_{2}}(u) \cdot d_{G_{1}+G_{2}}(v) .
$$

$$
\begin{aligned}
& =\sum_{u v \in E\left(S\left(G_{1}\right)\right)}\left[d_{G_{1}}(u)\left(2+n_{2}\right)\right]+\sum_{u v \in E\left(G_{2}\right)}\left[\left(d_{G_{2}}(u)+m_{1}\right)\left(d_{G_{2}}(v)+m_{1}\right)\right] \\
& u \in V\left(G_{1}\right) \\
& v \in V_{s}\left(G_{1}\right) \\
& +\sum_{u v \in E\left(G_{1}+G_{2}\right)}\left[\left(2+n_{2}\right)\left(d_{G_{2}}(v)+m_{1}\right)\right] \\
& u \in V_{s}\left(G_{1}\right) \\
& v \in V\left(G_{2}\right) \\
& =\left(2+n_{2}\right) \sum_{\substack{u v \in E\left(S\left(G_{1}\right)\right) \\
u \in V\left(G_{1}\right)}} d_{G_{1}}(u)+\sum_{u v \in E\left(G_{2}\right)}\left[d_{G_{2}}(u) d_{G_{2}}(v)+m_{1}\left(d_{G_{2}}(u)+d_{G_{2}}(v)\right)+m_{1}^{2}\right] \\
& v \in V_{s}\left(G_{1}\right) \\
& +\left(2+n_{2}\right) \sum_{u \in V_{s}\left(G_{1}\right)} 1 \sum_{v \in V\left(G_{2}\right)}\left(d_{G_{2}}(v)+m_{1}\right) \\
& =\left(2+n_{2}\right) \sum_{\substack{u v \in E\left(S\left(G_{1}\right)\right) \\
u \in V\left(G_{1}\right)}} d_{G_{1}}(u)+\sum_{u v \in E\left(G_{2}\right)} d_{G_{2}}(u) d_{G_{2}}(v)+m_{1} \sum_{u v \in E\left(G_{2}\right)}\left(d_{G_{2}}(u)+d_{G_{2}}(v)\right) \\
& v \in V_{s}\left(G_{1}\right) \\
& +m_{1}^{2} \sum_{u v \in E\left(G_{2}\right)} 1+\left(2+n_{2}\right) \sum_{u \in V_{s}\left(G_{1}\right)} 1\left(\sum_{v \in V\left(G_{2}\right)} d_{G_{2}}(v)+m_{1} \sum_{v \in V\left(G_{2}\right)} 1\right) \\
& =\left(2+n_{2}\right) \sum_{u v \in E\left(S\left(G_{1}\right)\right)} d_{G_{1}}(u)+M_{2}\left(G_{2}\right)+m_{1} M_{1}\left(G_{2}\right)+m_{1}^{2} m_{2} \\
& +m_{1}\left(2+n_{2}\right)\left(2 m_{2}+m_{1} n_{2}\right) \\
& =m_{1} M_{1}\left(G_{2}\right)+M_{2}\left(G_{2}\right)+m_{1}^{2} m_{2}+m_{1}\left(2+n_{2}\right)\left(2 m_{2}+m_{1} n_{2}\right)+\left(2+n_{2}\right) \\
& \sum_{u v \in E\left(S\left(G_{1}\right)\right)} d_{G_{1}}(u) \text {. }
\end{aligned}
$$

Theorem 6. Let $G_{1}=\left(n_{1}, m_{1}\right)$ and $G_{2}=\left(n_{2}, m_{2}\right)$ are two graphs; then, the first reformulated Zagreb index of subdivision vertex join $G_{1}+G_{2}$ is given as

$$
\begin{aligned}
& E M_{1}\left(G_{1} \dot{+} G_{2}\right)= \\
& 5 m_{1} M_{1}\left(G_{2}\right)+E M_{1}\left(G_{2}\right)+m_{1} n_{2}\left(n_{2}^{2}+m_{1}^{2}\right)+n_{2}^{2}\left(2 m_{1}^{2}+m_{1}^{\prime}\right) \\
& \quad+4 m_{1} m_{2}\left(2 m_{1}+n_{2}-2\right)+\sum_{u v \in E\left(S\left(G_{1}\right)\right)}\left[d_{G_{1}}(u)^{2}+2 n_{2} d_{G_{1}}(u)\right] .
\end{aligned}
$$


Proof. From the definition of the first reformulated Zagreb Now, we apply Lemma 2: index, we have

$$
E M_{1}\left(G_{1} \dot{+} G_{2}\right)=\sum_{u v \in E\left(G_{1}+G_{2}\right)}\left[d_{G_{1}+G_{2}}(u) \dot{+} d_{G_{1}+G_{2}}(v)-2\right]^{2} \text {. }
$$

$$
\begin{aligned}
& =\sum_{u v \in E\left(S\left(G_{1}\right)\right)}\left[d_{G_{1}}(u)+2+n_{2}-2\right]^{2}+\sum_{u v \in E\left(G_{2}\right)}\left[d_{G_{2}}(u)+m_{1}+d_{G_{2}}(v)+m_{1}-2\right]^{2} \\
& u \in V\left(G_{1}\right) \\
& v \in V_{s}\left(G_{1}\right) \\
& +\sum_{u v \in E\left(G_{1}+G_{2}\right)}\left[2+n_{2}+d_{G_{2}}(v)+m_{1}-2\right]^{2} \\
& u \in V_{s}\left(G_{1}\right) \\
& v \in V\left(G_{2}\right) \\
& =\sum_{\substack{u v \in E\left(S\left(G_{1}\right)\right) \\
u \in V\left(G_{1}\right)}}\left[d_{G_{1}}(u)+n_{2}\right]^{2}+\sum_{\substack{u \in E\left(G_{2}\right) \\
v \in V}}\left[\left(d_{G_{2}}(u)+d_{G_{2}}(v)-2\right)+2 m_{1}\right]^{2} \\
& u \in V\left(G_{1}\right) \\
& v \in V_{s}\left(G_{1}\right) \\
& +\sum_{\substack{u v \in E\left(G_{1}+G_{2}\right) \\
u \in V_{s}\left(G_{1}\right) \\
v \in V\left(G_{2}\right)}}\left[n_{2}+\left(d_{G_{2}}(v)+m_{1}\right)\right]^{2} \\
& =\sum_{\substack{u v \in E\left(S\left(G_{1}\right)\right) \\
u \in V\left(G_{1}\right)}}\left[d_{G_{1}}(u)^{2}+n_{2}^{2}+2 n_{2} d_{G_{1}}(u)\right]+\sum_{u v \in E\left(G_{2}\right)}\left[\left(d_{G_{2}}(u)+d_{G_{2}}(v)-2\right)^{2}\right. \\
& v \in V_{s}\left(G_{1}\right) \\
& +\left(2 m_{1}\right)^{2}+2\left(2 m_{1}\right)\left(d_{G_{2}}(u)+d_{G_{2}}(v)-2\right)+\sum_{\substack{u v \in E\left(G_{1}+G_{2}\right) \\
u \in V_{s}\left(G_{1}\right)}}\left[n_{2}^{2}+\left(d_{G_{2}}(v)+m_{1}\right)^{2}+2 n_{2}\left(d_{G_{2}}(v)+m_{1}\right)\right] \\
& u \in V_{s}\left(G_{1}\right) \\
& =\sum_{\substack{u v \in E\left(S\left(G_{1}\right)\right) \\
u \in V\left(G_{1}\right) \\
v \in V_{s}\left(G_{1}\right)}} d_{G_{1}}(u)^{2}+n_{2}^{2} \sum_{u v \in E\left(S\left(G_{1}\right)\right)} 1+2 n_{2} \sum_{\substack{u v \in E\left(S\left(G_{1}\right)\right) \\
u \in V\left(G_{1}\right) \\
v \in V_{s}\left(G_{1}\right)}} d_{G_{1}}(u)+\sum_{u v \in E\left(G_{2}\right)} d(e)^{2}+ \\
& 4 m_{1}^{2} \sum_{u v \in E\left(G_{2}\right)} 1+4 m_{1} \sum_{u v \in E\left(G_{2}\right)}\left(d_{G_{2}}(u)+d_{G_{2}}(v)\right)-8 m_{1} \sum_{u v \in E\left(G_{2}\right)} 1+n_{2}^{2} \sum_{u \in V_{s}\left(G_{1}\right)} 1 \\
& \sum_{v \in V\left(G_{2}\right)} 1+\sum_{u \in V_{s}\left(G_{1}\right)} 1 \sum_{v \in V\left(G_{2}\right)} d_{G_{2}}(v)^{2}+m_{1}^{2} \sum_{u \in V_{s}\left(G_{1}\right)} 1 \sum_{v \in V\left(G_{2}\right)} 1+2 m_{1} \sum_{u \in V_{s}\left(G_{1}\right)} 1 \\
& \sum_{v \in V\left(G_{2}\right)} d_{G_{2}}(v)+2 n_{2} \sum_{u \in V_{s}\left(G_{1}\right)} 1 \sum_{v \in V\left(G_{2}\right)} d_{G_{2}}(v)+2 m_{1} n_{2} \sum_{u \in V_{s}\left(G_{1}\right)} 1 \sum_{v \in V\left(G_{2}\right)} 1= \\
& \sum_{u v \in E\left(S\left(G_{1}\right)\right)}\left[d_{G_{1}}(u)^{2}+2 n_{2} d_{G_{1}}(u)\right]+m_{1}^{\prime} n_{2}^{2}+E M_{1}\left(G_{2}\right)+4 m_{1}^{2} m_{2}+4 m_{1} M_{1}\left(G_{2}\right) \\
& u \in V\left(G_{1}\right) \\
& v \in V_{s}\left(G_{1}\right) \\
& -8 m_{1} m_{2}+m_{1} n_{2}^{3}+m_{1} M_{1}\left(G_{2}\right)+m_{1}^{3} n_{2}+4 m_{1}^{2} m_{2}+4 m_{1} m_{2} n_{2}+2 m_{1}^{2} n_{2}^{2} \\
& =5 m_{1} M_{1}\left(G_{2}\right)+E M_{1}\left(G_{2}\right)+m_{1} n_{2}\left(n_{2}^{2}+m_{1}^{2}\right)+n_{2}^{2}\left(2 m_{1}^{2}+m_{1}^{\prime}\right) \\
& +4 m_{1} m_{2}\left(2 m_{1}+n_{2}-2\right)+\sum_{u v \in E\left(S\left(G_{1}\right)\right)}\left[d_{G_{1}}(u)^{2}+2 n_{2} d_{G_{1}}(u)\right] \text {, }
\end{aligned}
$$


which is our required result.

\section{Conclusion}

In this study, we proposed two new variants of special graph products and found their exact expressions for the first Zagreb index, second Zagreb index, and first reformulated Zagreb index. These new graph invariants can be used to construct come cellular networks, for example, the factor cellular network or some hybrid cellular networks. In future, other degree and distance-based topological indices of these graph operations can be found. The obtained results may help to construct and investigate the topological indices of complex networks structures.

\section{Data Availability}

No data were used to support this study.

\section{Conflicts of Interest}

The authors declare that they have no conflicts of interest.

\section{References}

[1] E. A. Bender and S. G. Williamson, Lists, Decisions and Graphs, Vol. 148, University of California, , San Diego, CA, USA, 2010.

[2] N. Deo, Graph Theory with Applications to Engineering and Computer Science, Prentice Hall, Hoboken, NJ, USA, 1990.

[3] I. Gutman and N. Trinajstić, "Graph theory and molecular orbitals. Total $\varphi$-electron energy of alternant hydrocarbons," Chemical Physics Letters, vol. 17, no. 4, pp. 535-538, 1972.

[4] B. Bollobás and P. Erdös, "Graphs of extremal weights," Ars Combinatori, vol. 50, pp. 225-233, 1998.

[5] J. Devillers and A. T. Balaban, Topologicial Indices and Related Descriptors in QSAR and QSPR, Gordon and Breach, Amsterdam, Netherlands, 1999.

[6] I. Gutman and K. C. Das, "The first Zagreb index 30 years after," MATCH Communications in Mathematical and in Computer Chemistry, vol. 50, pp. 83-92, 2004.

[7] K. C. Das and I. Gutman, "Some properties of of the second Zagreb index," MATCH Communications in Mathematical and in Computer Chemistry, vol. 52, pp. 103-112, 2004.

[8] R. Frucht and F. Harary, "On the corona of two graphs," Aequationes Mathematicae, vol. 4, no. 3, pp. 322-325, 1970.

[9] Y. Gao, M. Imran, M. R. Farahani, and H. M. A. Siddiqui, "Some connectivity indices and Zagreb index of honeycomb graphs," International Journal of Pharma Sciences and Research, vol. 9, no. 5, pp. 2080-2087, 2018.

[10] I. Gutman, M. K. Jamil, and N. Akhter, "Graphs with fixed number of pendent vertices and first Zagreb index," Transactions on Combinatorics, vol. 4, pp. 43-48, 2015.

[11] A. Milićević, S. Nikolić, and N. Trinajstić, "On reformulated Zagreb indices," Molecular Diversity, vol. 8, pp. 393-399, 2004.

[12] V. Yegnanarayanan, P. R. Thiripurasundari, and T. Padmavathy, "On some graph operations and related applications," Electronic Notes in Discrete Mathematics, vol. 33, pp. 123-130, 2009.

[13] P. Lu and Y. Miao, "Spectra of the subdivision-vertex and subdivision-edge corona," 2013, http://arxiv.org/abs/1302. 0457.
[14] S. Barik and G. Sahoo, "On the laplacian spectra of some variants of corona," Linear Algebra and its Applications, vol. 512, 2017.

[15] M. H. Khalifeh, H. Yousefi-Azari, and A. R. Ashrafi, "The first and second Zagreb indices of some graph operations," Discrete Applied Mathematics, vol. 157, no. 4, pp. 804-811, 2009.

[16] K. C. Das, A. Yurttas, M. Togan, A. S. Cevik, and I. N. Cangul, "The multiplicative Zagreb indices of graph operations," Journal of Inequalities and Applications, vol. 2013, no. 1, p. 90, 2013.

[17] M. Azari and A. Iranmanesh, "Chemical graphs constructed from rooted product and their Zagreb indices," MATCH Communications in Mathematical and in Computer Chemistry, vol. 70, pp. 901-919, 2013.

[18] M. K. Jamil and I. Tomescu, "First reformulated Zagreb index and some graph operations," Ars Combinatoria, vol. 138, pp. 193-209, 2018.

[19] H. M. A. Siddiqui, S. Baby, M. F. Nadeem, and M. K. Shafiq, "Bounds of some degree based indices of lexicographic product of some connected graphs," Polycyclic Aromatic Compounds, 2020.

[20] A. R. Ashrafi, T. Došlić, and A. Hamzeh, "The Zagreb coindices of graph operations," Discrete Applied Mathematics, vol. 158, no. 15, pp. 1571-1578, 2010.

[21] M. Arezoomand and B. Taeri, "Zagreb indices of the generalized hierarchical product of graphs," MATCH Communications in Mathematical and in Computer Chemistry, vol. 69, no. 1, pp. 131-140, 2013.

[22] M. Eliasi and B. Taeri, "Four new sums of graphs and their Wiener indices," Discrete Applied Mathematics, vol. 157, no. 4, pp. 794-803, 2009.

[23] B. Basavanagoud and S. Patil, "The hyper-Zagreb index of four operations on graphs," Mathematical Sciences Letters, vol. 6, no. 2, pp. 193-198, 2017.

[24] J. B. Babujee and S. Ramakrishnan, "Topological indices and new graph structures," Applied Mathematical Sciences, vol. 6, no. 108, pp. 5383-5401, 2012.

[25] K. Kiruthika, "Zagreb indices and Zagreb coindices of some graph operations," International Journal of Advanced Research in Engineering and Technology, vol. 7, no. 3, pp. 25-41, 2016.

[26] G. H. Shirdel, H. Rezapour, and A. M. Sayadi, "The hyperZagreb Index of graph operations," Iranian Journal of Mathematical Chemistry, vol. 4, no. 2, pp. 213-220, 2013

[27] N. De, A. Pal, and S. M. A. Pal, "The F-coindex of some graph operations," SpringerPlus, vol. 5, no. 1, p. 221, 2016.

[28] N. De, "Computing f-index of different corona products of graphs," Bulletin of Mathematical Sciences and Applications, vol. 19, pp. 24-30, 2017. 